Supporting Information

\title{
Bimorph Silk Microsheets with Programmable Actuating Behavior: Experimental Analysis and Computer Simulations
}

\author{
Chunhong Ye, ${ }^{a}$ Svetoslav V. Nikolov, ${ }^{b}$ Ren D. Geryak,, ${ }^{a}$ Rossella Calabrese, ${ }^{c}$ John F. \\ Ankner, ${ }^{d}$ Alexander Alexeev, ${ }^{b}$ David L. Kaplan, ${ }^{c}$ Vladimir V. Tsukruk ${ }^{a,}$ \\ a School of Materials Science and Engineering, Georgia Institute of Technology, Atlanta, \\ Georgia 30332 (USA) \\ ${ }^{b}$ School of Mechanical Engineering, Georgia Institute of Technology, Atlanta, Georgia 30332 \\ (USA) \\ ${ }^{c}$ Department of Biomedical Engineering, Tufts University, 4, Colby street, Medford, MA 02155 \\ USA \\ d Spallation Neutron Source, Oak Ridge National Laboratory, Oka Ridge, Tennessee 37831, \\ USA
}

"Corresponding author. Email: Vladimir@mse.gatech.edu 


\section{Supplementary Materials}

\section{Silk-on-silk microsheet fabrication summary}

Table S1 Summary of the silk based microsheets explored in the experiments.

\begin{tabular}{|c|c|c|c|c|c|c|c|c|}
\hline \multirow{2}{*}{ Figure } & \multirow{2}{*}{$\begin{array}{c}\text { Microsheet } \\
\text { apect ratio } \\
\text { (width: } 50 \mu \mathrm{m})\end{array}$} & \multirow{2}{*}{ Microsheet composition } & \multicolumn{3}{|c|}{ Layer thickness/ nm } & \multicolumn{2}{|c|}{ Thickness ratio } & \multirow{2}{*}{$\begin{array}{l}\text { Explored } \\
\text { factor }\end{array}$} \\
\hline & & & PS Prelayer & $\begin{array}{c}\text { Silk ionomer } \\
\text { layer (SI layer) }\end{array}$ & $\beta$-sheets & $\begin{array}{c}\beta \text {-sheets/SI } \\
\text { layer }\end{array}$ & PS/SI layer & \\
\hline Fig. 5a & \multirow{2}{*}{$1: 2$} & PS-(SF-PG/SF-PL) ${ }_{12}$-( $(\beta \text {-sheet })_{1}$ & 88.4 & 41.6 & 6.8 & 0.16 & 2.12 & \multirow{2}{*}{$\begin{array}{l}\text { Controllabe } \\
\text { Rolling } \\
\text { process }\end{array}$} \\
\hline Fig. 5b & & PS-(SF-PG/SF-PL $)_{12}-(\beta \text {-sheet })_{10}$ & 68.2 & 49.5 & 40.4 & 0.82 & 1.38 & \\
\hline Fig. $6 a$ & \multirow{5}{*}{$1: 2$} & \multirow{5}{*}{ PS-(SF-PG/SF-PL) ${ }_{12}$-( $(\beta \text {-sheet })_{10}$} & 207.3 & \multirow{5}{*}{$48.5 \pm 2.5$} & \multirow{5}{*}{$34.5 \pm 4.5$} & \multirow{5}{*}{$0.71 \pm 0.06$} & 3.91 & \multirow{5}{*}{$\begin{array}{l}\text { varied PS } \\
\text { prelayer } \\
\text { thickness }\end{array}$} \\
\hline Fig. 6b & & & 110.0 & & & & 2.33 & \\
\hline Fig. $6 \mathrm{c}$ & & & 95.0 & & & & 1.95 & \\
\hline Fig. $6 \mathrm{~d}$ & & & 68.2 & & & & 1.38 & \\
\hline Fig. $6 e$ & & & 20.0 & & & & 0.45 & \\
\hline Fig. $8 a$ & $1: 1$ & \multirow{4}{*}{ PS-(SF-PG/SF-PL) ${ }_{12}$-( $(\beta \text {-sheet })_{10}$} & \multirow{4}{*}{$97.3 \pm 8.7$} & \multirow{4}{*}{$50.5 \pm 2.0$} & \multirow{4}{*}{$34.2 \pm 5.0$} & \multirow{4}{*}{$0.68 \pm 0.09$} & \multirow{4}{*}{$1.71 \pm 0.53$} & \multirow{4}{*}{$\begin{array}{l}\text { varied } \\
\text { pattern } \\
\text { aspect ratio }\end{array}$} \\
\hline Fig. 8b & $1: 2$ & & & & & & & \\
\hline Fig. 8c & $1: 4$ & & & & & & & \\
\hline Fig. 8d & $1: 8$ & & & & & & & \\
\hline Fig. 10a & \multirow{4}{*}{$1: 2$} & PS-(SF-PG/SF-PL) ${ }_{12}-(\beta \text {-sheet })_{1}$ & \multirow{4}{*}{$81.6 \pm 4.5$} & \multirow{4}{*}{$44.8 \pm 5.2$} & 6.8 & 0.16 & \multirow{4}{*}{$1.84 \pm 0.26$} & \multirow{4}{*}{$\begin{array}{l}\text { varied } \beta- \\
\text { sheet } \\
\text { thickness }\end{array}$} \\
\hline Fig. 10b & & PS-(SF-PG/SF-PL) ${ }_{12}-(\beta-\text { sheet })_{7}$ & & & 25.2 & 0.56 & & \\
\hline Fig. 10c & & PS-(SF-PG/SF-PL $)_{12}-(\beta \text {-sheet })_{9}$ & & & 31.6 & 0.77 & & \\
\hline Fig. 10d & & PS-(SF-PG/SF-PL) ${ }_{12}-(\beta \text {-sheet })_{13}$ & & & 52.9 & 1.01 & & \\
\hline
\end{tabular}

*The text in red indicated the varied parameter in corresponding the figure.

\section{Theoretical Analysis}

In order to analyze the deformation of the tri-layered structures we modified a thermomechanical model for bending in composite sections. ${ }^{1}$ In doing this, we assumed that $\alpha T \approx \varepsilon$. Where $\alpha T$ is the thermal expansion and $\varepsilon$ is the experimental swelling ratio due to changes in the $\mathrm{pH}$. The cross-sectional view of the composite network is shown in Figure S1. In this Figure, $2 h_{1}, 2 h_{2}$, and $2 h_{3}$ represent the thickness of the polystyrene, silk-ionomer, and $\beta$-sheet layers, respectively. Meanwhile, $h_{i, j}$ represents the distance between the centroidal lines of the $i$ and $j$ layers. The final radius of curvature of the composite structure can be predicted by considering the mechanical and swelling stresses in each layer. Doing this the radius can be defined as:

$$
r=\frac{E_{1} I_{1}+E_{2} I_{2}+E_{3} I_{3}+\operatorname{abs}\left(\bar{y}\left(h_{12} E_{1} A_{1}-h_{23} A_{3} E_{3}\right)\right)+h_{12} h_{23} A_{3} E_{3}}{\left[h_{12} E_{1} A_{1}-h_{23} A_{3} E_{3}\right] \bar{\varepsilon}}
$$


Here $E_{i}, I_{i}$ and $A_{i}$ are the corresponding moduli of elasticity, area moments of inertia, and cross-sectional areas.

Also:

$$
\bar{y}=\frac{E_{2} A_{2} h_{12}+E_{3} A_{3} h_{13}}{E_{1} A_{1}+E_{2} A_{2}+E_{3} A_{3}}
$$

And:

$$
\bar{\varepsilon}=\frac{E_{2} A_{2} \varepsilon}{E_{1} A_{1}+E_{2} A_{2}+E_{3} A_{3}}
$$

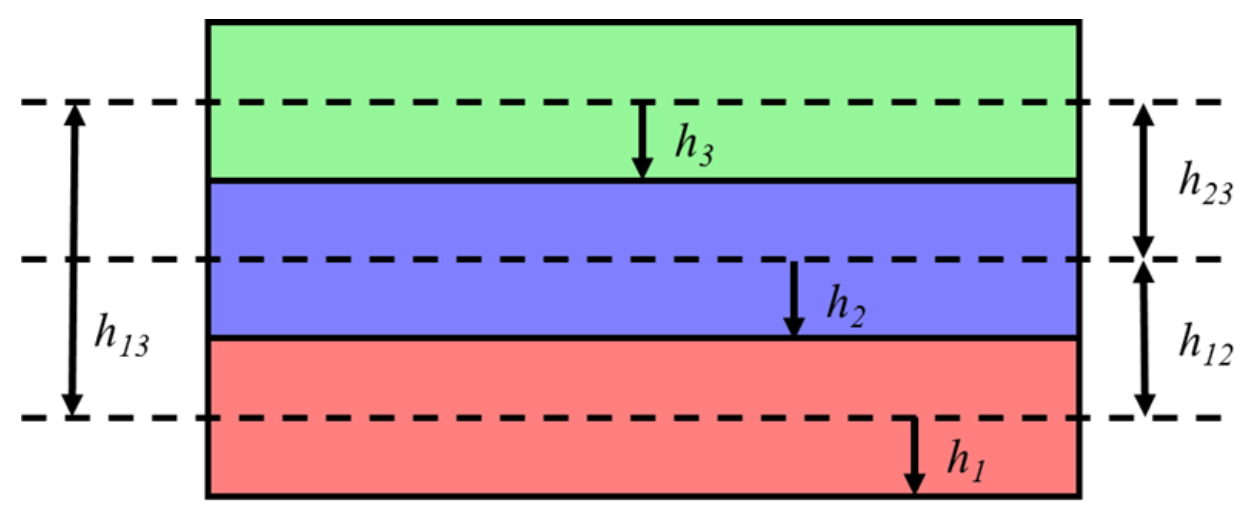

Figure S1. Cross-sectional view of composite structure. Green layer represents beta-sheet, blue layer represents the silk-ionomer, and red layer represents the polystyrene

Using the scaling outlined in Equation 1-3, we can study how the final radius changes when we vary the $\beta$-sheet and silk ionomer layer thicknesses, and more importantly how these changes compare to experiments. Figure $\mathrm{S} 2$ shows how the radius changes as a function of PS thickness for five different $\beta$-sheet thicknesses. Here, the change in sign of the radius represents a switch in the folding direction. Negative values of the radius correspond to folding on the $\beta$-sheet side and positive values represent folding on the PS side. The dotted lines represent the PS thicknesses for which a switch in the folding direction occurs. From the figure it can be seen that as the $\beta$-sheet thickness is increased the dotted lines shift to the right, indicating the fact that as we increase the $\beta$ - 
sheet thickness thicker PS layers are required to produce the switch in folding. When the $\beta$-sheet thickness is $34.4 \mathrm{~nm}$ the theory captures the experimental trend. The predicted $\beta$-sheet thickness also matches the experimental measurements for the $\beta$ sheet layer $(33.5 \pm 0.9 \mathrm{~nm})$.

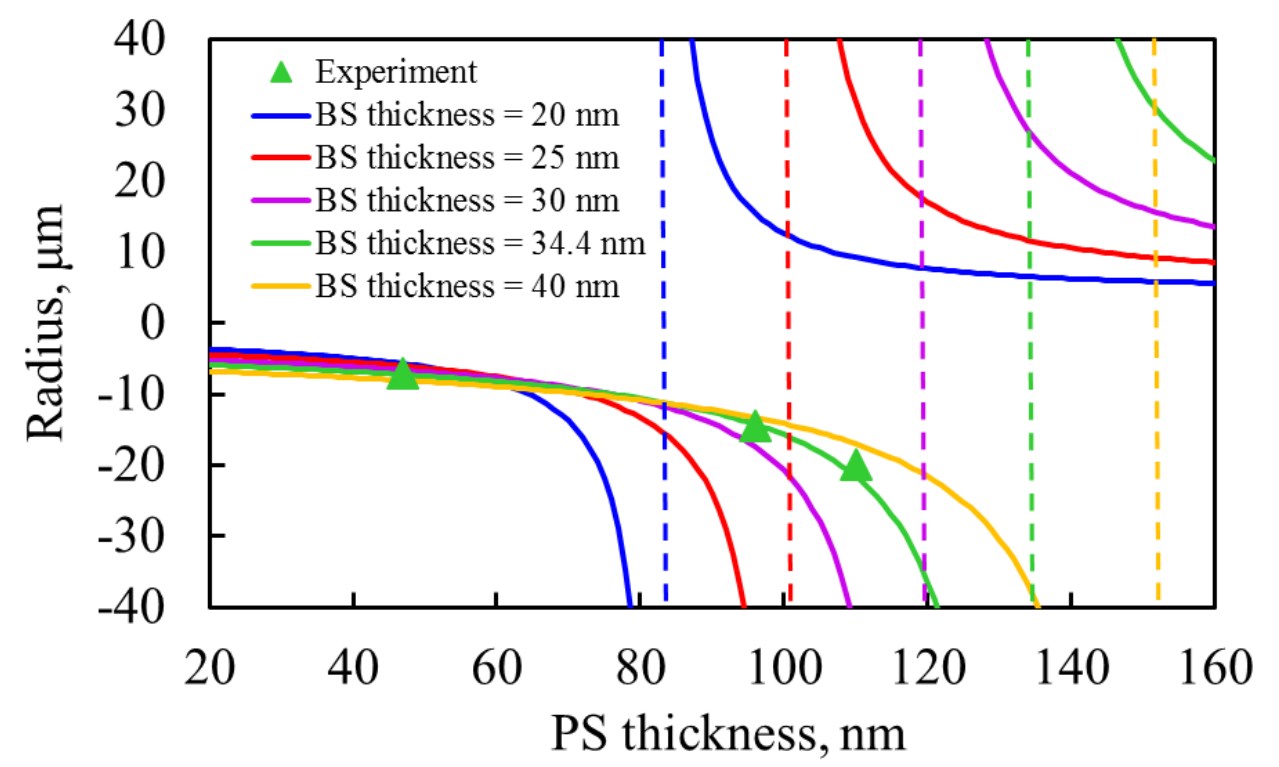

Figure S2. Plot showing how radius changes as a function of the PS thickness for different $\beta$ sheet thicknesses. For composite network we assumed a PS stiffness of $1.8 \mathrm{GPa}$, silk ionomer layer thickness of $388 \mathrm{~nm}$ and stiffness of $11 \mathrm{MPa}$, and $\beta$-sheet stiffness of $8.6 \mathrm{GPa}$.

Similar analysis can be applied as we vary the thickness of silk ionomer layer. A plot showing how the radius changes as a function of the PS thickness for different silk ionomer thicknesses is shown in Figure S3. From the figure it can be seen that as the silk ionomer layer thickness is increased, the dotted lines (indicating the switch in folding direction) again shift to the right. To fully understand this effect we have to think of the limiting case when the silk ionomer layer thickness is very larger. In this case, the thickness of the PS and $\beta$-sheet layers is much smaller than the thickness of the silk ionomer layer and so we can think of each passive layer (PS layer and $\beta$-sheets) as a spring $(F=k x)$.

For the limiting case when the silk ionomer thickness is very large. In this special case, the thickness of the passive layers is not important and the stiffness is solely determined by the modulus of each layer. The shift in the dotted lines in Figure S3 represents the transition to this limiting case. From the figure we see that the theory 
predicts a silk ionomer layer thickness of $388 \mathrm{~nm}$. Experimentally, the swollen thickness of the silk ionomer layer is $\sim 380 \mathrm{~nm}$. As such we can see that there is a nice agreement between scaling and experiments.

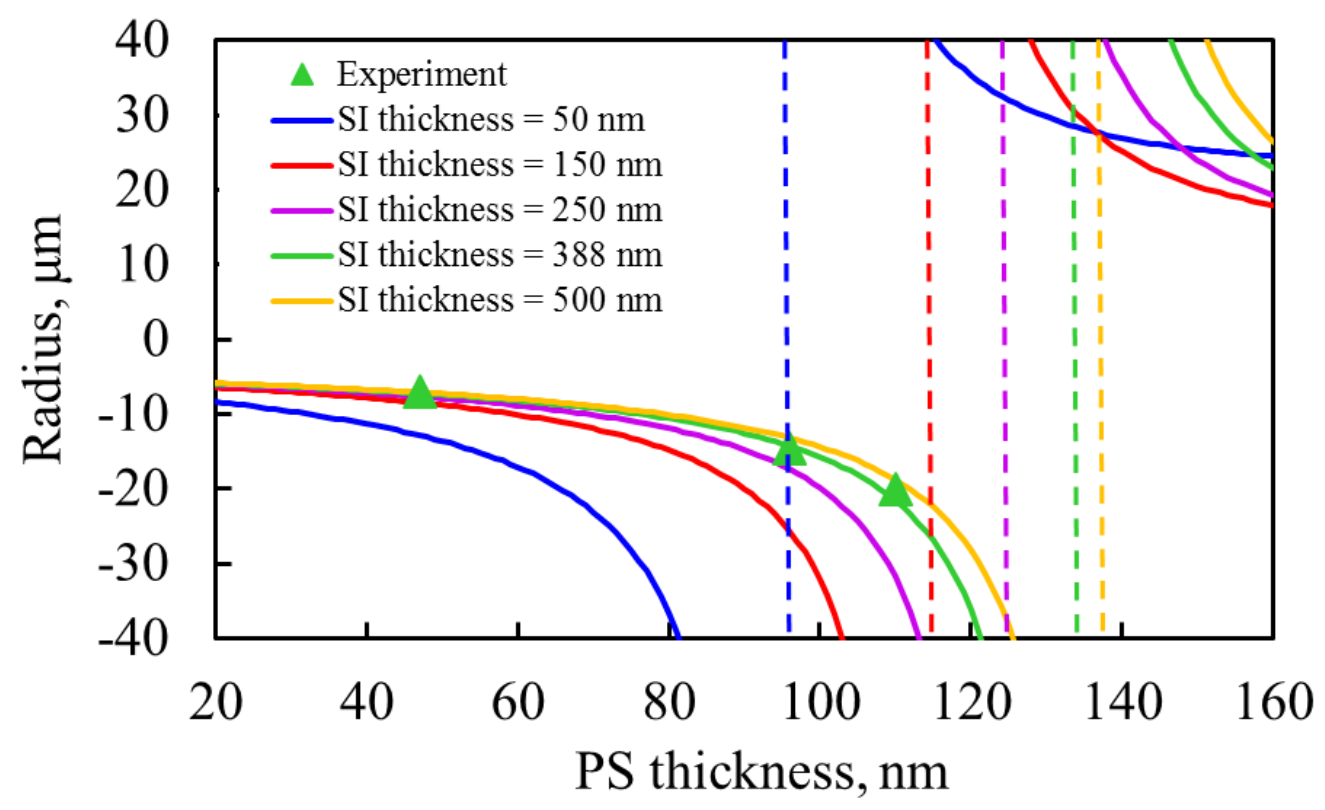

Figure S3. Plot showing how radius changes as a function of PS thickness for different silk ionomer layer thicknesses. Here we assume $\beta$-sheet thickness of $34.4 \mathrm{~nm}$ and stiffness of 8.6 $\mathrm{GPa}$, PS stiffness of $1.8 \mathrm{GPa}$,

\section{Lattice spring model simulations}




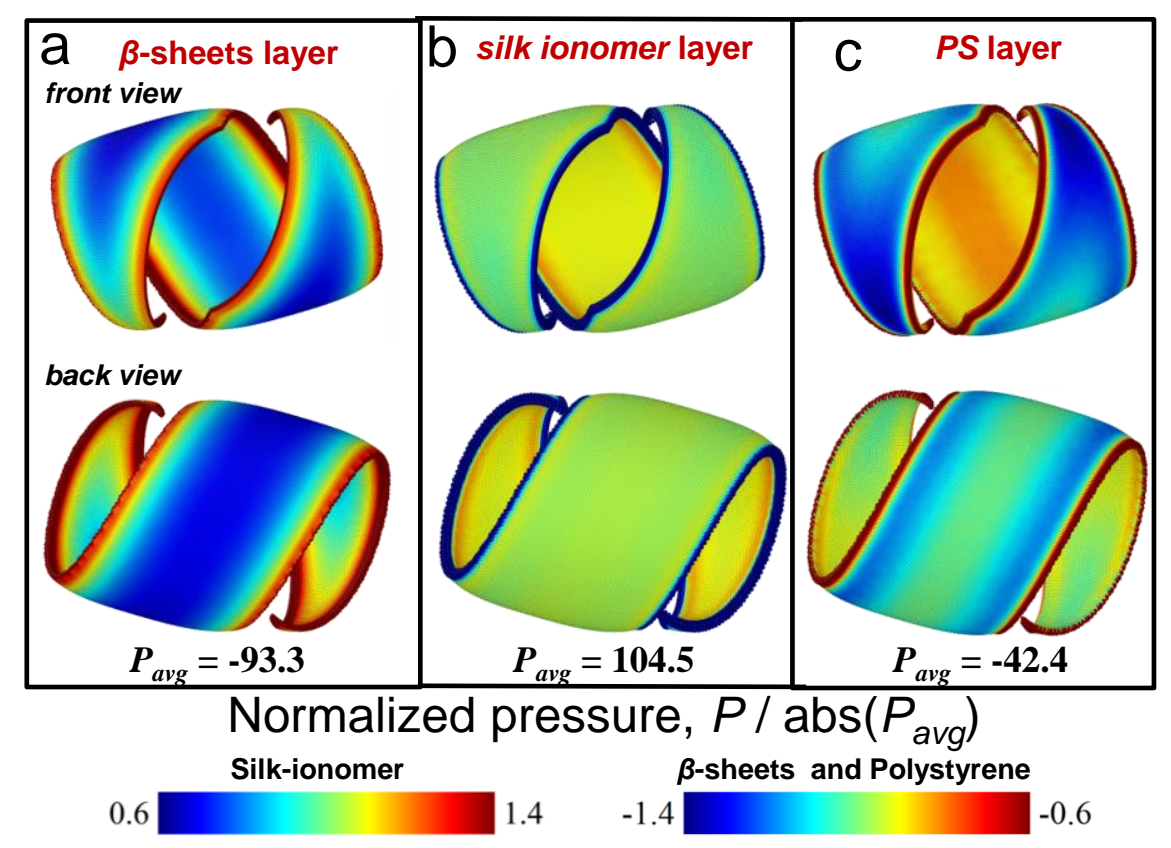

Figure S4. Stress map obtained from computational simulation for each individual lays within the sandwiched $30^{\circ}$ parallelograms silk microsheet. a) $\beta$-sheet; b) silk ionomer layer; c) PS layer. Top row indicated front view and the bottom row is the back view for each panel.

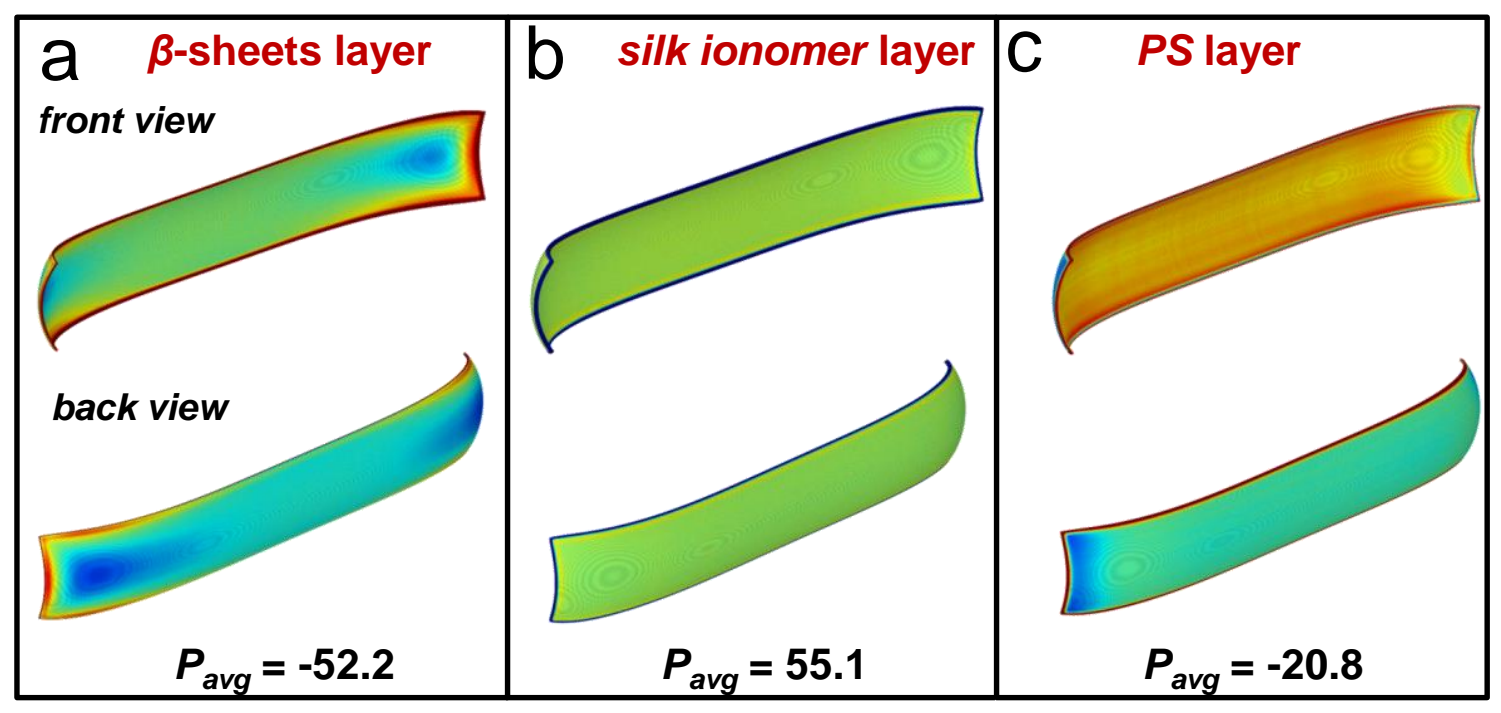

Normalized pressure, $P /$ abs $\left(P_{\text {avg }}\right)$

Silk-ionomer

0.6

1.4 $\beta$-sheets and Polystyrene

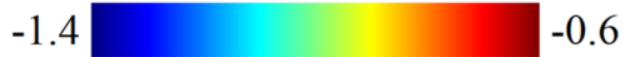

Figure S5. Stress map obtained from computational simulation for each individual lays within the sandwiched silk microsheet with an aspect ratio of 1:8. a) $\beta$-sheet; b) silk ionomer layer; c) PS layer. Top row indicated front view and the bottom row is the back view for each panel. 


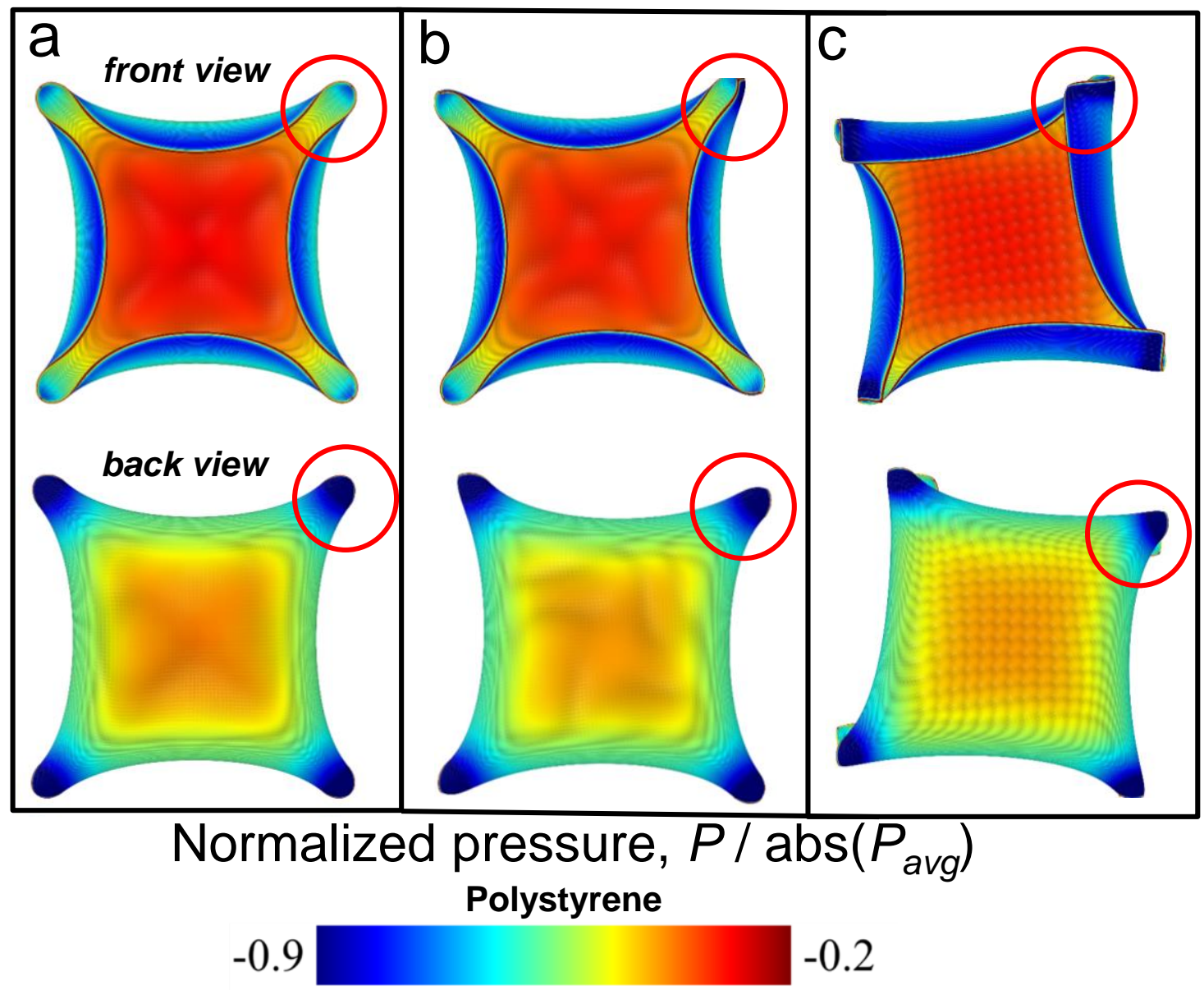

Figure S6. Color map of calculated stress within the PS layer as an example in the sandwiched silk microsheets with an aspect ratio of 1: 1 over a time series (a-c) (from left to right). Front views as shown in top row and back views in the bottom row.

[1] Nayfeh, A. H.; Baker, S. R. Thermomechanical Distortion of Composite Panels. Fiber Sci. and Tech. 1977, 10, 139-149. 